UDC 381.324

\title{
THE SELF-ORGANIZING RADIO NETWORKS AND ULTRA WIDE BAND SIGNALS
}

\author{
Sergey G. Bunin \\ Institute of Telecommunication Systems, National Polytechnic University, Kyiv, Ukraine
}

\begin{abstract}
The paper proposes to use impulse radio ultra wide band signals in self-organizing ad hoc and MANET networks to solve multiple access and widen networks coverage area problems. New methods of signals reception and information capacity increasing are proposed.
\end{abstract}

Keywords—ad hoc, MANET, IR-UWB, multiple access, frequency band, "non energy" reception method, information.

\section{Introduction}

For the last decade, many researchers and developers have dedicated themselves to self-organizing radio networks - the systems of radios-terminals with mutual multihop relaying and routing. Networks are the random structure, which consists of either many stationary (ad hoc) or mobile (MANET) subscribers, united only under the general rules (protocols) of interaction. It is assumed that such networks, as a rule, do not use any stationary infrastructure, but they can organize their dynamically changing hierarchy for an improvement of network functioning by using these or other tacticaltechnical characteristics of terminals - larger radio transmitter's power or the power capacity of batteries, directional antennas, preferential location which ensures larger zone of radio visibility, etc. Some hierarchical relations between terminals also can be used.

Actually, such networks must replace the portable "walky-talky" telephone radio networks by automating the process of connecting subscribers and widen forms of information (data, photos, video in digital forms).

The self-organizing radio networks, gathered from the random radio stations, appeared in the 70's of past century and they were "invented" by radio amateurs. Using AX.25 packet switching protocol, wireless enthusiasts sent information for the certain recipient by transferring it to its first-encountered radio station, equipped with packet radio communication means. After receiving the message in many packets form, the radio station is attempted to find the addressee of the message or, at least, any station, which is located in the country or on the continent of the addressee. For routing the station call-signs, containing symbols of countries, were used.

Another example of the ad hoc network is Internet! Internet was created and still develops as the network of the arbitrarily packet routers, which provides mutual relaying of packets for many subscribers using domain names. The switching on and disconnection of various routers in the network does not disrupt the connectivity of final subscribers, but only changes ways of packets routs in the network.

\section{Ad hoc and MANET niches}

The expenditure of material and temporary resources for research and developing of self-organizing radio networks obviously assumes the prognostication of the "niche" for their use among the sufficiently developed contemporary systems of stationary and mobile radio communication. In our view, the basic fields of application are the following:

- Military tactical radio communication, which ensures the high networks survivability due to decentralization and mutual multiple connectivity;

- Departmental or private networks (dispatch, industrial networks, temporary networks during the mass events - conferences, sport competitions, public meetings, etc.);

- Connections in groups of people and population which are away from the infrastructural communication networks (members of expeditions, remote agricultural areas, construction sites); 
- Communication during natural and technological catastrophes, when the infrastructure of regular communication is destroyed;

- Sensor networks for the information transmission from the mass of data sources - security systems, sensors of states and results of the measurements from different objects, development of the "clever houses", etc.

Among many problems, solved with the development of those self-organizing networks, there is one main thing - guarantee of reliable routing in the unstable network structure within a limited time.

Routing in the nonstationary topology and the subscriber activity is solved by some rules (protocols). Hundreds of routing protocols are proposed at present. They are subdivided into three basic types - proactive, reactive and hybrid.

Proactive protocols imply "knowledge" by each of the net terminals a number of subscribers in network and ways to reach to them. For this each switched in network terminal must gather the maximum amount of information about the state of network. These data are represented in the form the routing tables to the subscriber needed and number of necessary relay hops to reach it. It is understandable that such protocols work well in the stationary networks or the networks with the low-mobility topology - ad hoc. They require the significant time expenditure for the composition of tables by observation of the data exchange by the subscribers of the network or collect messages received in return to the subscriber inquiring requests.

Reactive protocols assume transfer by the subscribers of the inquiring messages about the presence of necessary subscriber in the network and the possible ways of access to it at time of request. These protocols are more suitable for the dynamically changing situation in the network, for example, in the networks of mobile terminals - MANET.

Hybrid protocols are developed for a compromise in the volumes of service and useful information traffic circulating in the self-organizing networks.

These protocols solve the problems of routing with more or less success, which depends on many factors, but in essence on the rate of changes in network topology.

\section{How many ad hoc and MANET networks are in use?} Why?

Answer to this question is such - practically no one.

The reason is a problem of avoiding or minimizing mutual interference when many terminals transmit at the same time.
Radio channel is the broadcasting medium. The signal of one subscriber is "audible" by all other subscribers located within its radio visibility zone. On the one hand this is positive property which makes possible data transmission to any of the surrounding terminals. But, on the other hand, transmission of two or more radio signals at the same time leads to interferences resulting in mutual data distortions on reception. This takes place when "classical" signals with sinusoidal carriers are used. Frequency selection of such signals assumes monopoly usage of the frequency by one user at any time. If the same frequency band is used by many users, time division must be used to prevent packets overlapping.

The wide band spectrum signals (CDMA) allow overlapping of several signals with equal signal levels at receiver input. The number of signals which coincide in the time is determined by the degree of their mutual orthogonality. The orthogonality usually depends on selected band spread sequences and signal base - the ratio of the bandwidth of the transmitted signal to the bandwidth of transmitted data. With an increase of data rates and limitations of available frequency bands, it is difficult to ensure the interference immunity of a more-less significant quantity of simultaneously transmitted signals. And equality of signal strength at receiver's inputs in radio networks is difficult or impossible to achieve.

The problem is usually solved by medium access control (MAC) protocols usage.

MAC protocols for multiple access are rules for transmission of each network subscriber taking into consideration needs of communication channel occupation by other subscribers. All multiple access protocols introduce mitigation of the network throughput and increase of transmissions delays due to packets necessity to stay in a line waiting for transmission. In ad hoc/MANET networks with many relayings the multiple access protocols must be carried out on each relaying terminal. Therefore, delays grow with increase of traffic generated by subscribers in the areas of mutual radio visibility and numbers of relayings on the route. The sum of delays can exceed the allowed data delivery time value, thus, making the network not suitable for the information transfer in a real time.

Thus, the fulfillment of multiple access protocols rule - one signal in the channel at any time, is a basic obstacle in the creation of productive self-organizing radio networks. There are dozens of MAC protocols using different mechanisms to solve the task to have just one signal on the channel at any time. But it is difficult if it is generally possible to use them in ad hoc/MANET networks because of many reasons - different distances between terminals and different propagation delays, dif- 
ferent signal to noise and interference ratio, interference from "hidden" terminals, etc.

This problem can be solved if overall traffic on the communication channel is much less then possible channel throughput. In this case one can use uncontrolled access of the "unslotted ALOHA" protocol with up to 18 percent of the channel maximum throughput. This means ineffective use of the allocated frequency resource.

Thus, the multiple access protocol problem makes high-performance ad hoc and MANET networks practically unrealizable.

\section{Impulse Radio Ultra Wide Band and ad hoc/MANET networks}

Impulse radio ultra wide band (IR -UWB) signals possess a whole series of the properties, which favorably compared them with signals based on sinusoidal carriers [2]:

- They perform effective current to electromagnetic fields transform;

- Good penetration through various propagation mediums;

- Low spectrum density which prevents interference to existing radio services and systems;

- These signals make it possible to locate source of transmission;

- It is possible to provide sharp antenna radiation pattern;

- Transmit-receive equipment can be realized mostly on digital technology.

Their use in self-organizing radio networks can solve the problems which prevent their wide practical implementation. First of all, noted above multiple access problem.

The transmission of information using these signals in radio networks is accomplished by sending blocks of ultra short impulses (usually less than 1 nanosecond duration) series. These blocks are impulse position code sequences (IPCS) which represent both transmitted symbol and the address of destination terminal (Fig. 1).

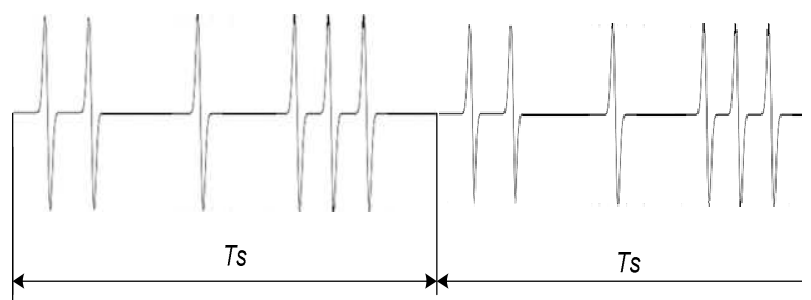

Fig. 1. Symbols represented by IR-UWB signals.
Sequences of impulses with the large averaged duty factor (ratio of the total pauses between impulses to the total impulses duration) must be used in the network. Such discrete signals make it possible to create large ensembles of orthogonal or quasi-orthogonal signals with minimal mutual interference. As a result, this permits to use an uncontrolled access to a communication channel - so-called simple ALOHA multiple access protocol. Even partly coincidences in the time impulses from different signals do not worsen the quality of each other, but improve it thanks to mutual increase the impulse power of the impulses. As opposed to CDMA, difference in impulse power from various terminals does not play significant role on received signals quality.

\section{FCC limit and long range communication}

The Federal Communication Commission of the USA in 2002 has made constrains on use of IR-UWB signals [3]. The Commission has limited the signals power by the value of $-41 \mathrm{dBm} / \mathrm{MHz}$ and the frequency band of 3,1 - 10,6 GHz. These limits were doubled in European Community countries. The limits were introduced to prevent mutual interference with other services using the same band. Thus, such small power limits the distance of communications up to several meters. The bandwidth limit does not permit to use impulses shorter than 1 nanosecond because ultra short impulses, passing this $7.5 \mathrm{GHz}$ band pass filter, become radio impulses with significantly longer durations. This means worsening such characteristics as transmission rates, tolerable numbers of simultaneously active subscribers in the network.

It is obvious that transmitters in ad hoc and MANET radio networks must have substantially more power and bandwidth to provide reliable communication in wide area networks. To make it possible a new frequency band outside widely used frequencies should be allocated for high rate long distance ad hoc/MANET services. Frequencies above $60 \mathrm{GHz}$ could be suitable for this purpose.

After some studies of suitable frequencies [4], it seems to us that $70-170 \mathrm{GHz}$ band could be applicable for this purpose. Atmospheric signal losses in this band do not exceed $12-15 \mathrm{~dB} / \mathrm{km}$. The durations of the impulses, which ensure the maximum of spectral density location in this frequency band, equal to 6 - 12 picoseconds values (picosecond $=10^{-12} \mathrm{~s}$ ). Generators of such ultra short impulses can be realized by contemporary impulse techniques.

Wide frequency band allows, on the one hand, using ultra short impulses without their duration increase, 
and, on the other hand, reducing spectral power densities, thus minimizing possible interferences to other services. Besides the displacement of IR-UWB signals spectrum into the band higher than $60 \mathrm{GHz}$ makes it possible to use high-pass filters both in transmitters and receivers to cut frequencies lower than this frequency, reducing interferences to and from common radio services on lower frequencies.

\section{Reception method}

The ultra short impulses bear very small energy because of their short duration. Signal energy $E=n P_{i} \tau$, where $P_{i}$-impulse power, $\tau$ - impulse duration, $n-$ number of impulses in the signal. The probability of error is determined by known relation $E / N_{0}\left(N_{0}\right.$ - noise spectral density at reception point) and also depends on type of modulation and reception method. Impulse position code modulation with identical impulse power in the signal sequence is the equivalent of the amplitude modulation, resulted in the worst reception effectiveness. However, only incoherent method is practically possible to receive ultra short impulses. Coherent method supposes comparison of received and reference waveforms in the correlator and requires precise synchronization. With the impulses duration less than the nanosecond, the accuracy of synchronization must be several times higher, which is difficult to carry out in portable devices and presence of Doppler shifts during motions. Therefore, incoherent method is the basic method of reception.

The incoherent method of the impulse position code reception is usually achieved with a circuit which is an equivalent of the matched filters made on delay lines (passive correlators). They are invariant to signal arrival moments, i.e., they do not require synchronization.

However, realization of this method meets difficulties in treating the ultra short impulses, because of their low energy and needs of accuracy in making analog delay line taps (not to mention broadband of the line) or needs to have a very high frequency clock for an equivalent digital processing circuit if one is used.

We have proposed a method and devices of socalled "non energy" impulse radio ultra wide band reception [5]. The idea of the method is to use all ultra short impulses level of which exceeds $r m s$ value of incoming noise as a trigger for monostable multivibrator. In response the multivibrator generates impulses of larger duration and necessary amplitude. These impulses are further processed by a digital system for signal selection and symbols decoding. Signal processing of longer duration impulses can be accomplished by software devices. A version of analog equivalent of the receiver is shown on fig. 2 . Thus, this approach of impulse selection rather than their energy collection significantly simplifies IR-UWB receivers. The noise immunity (sensitivity) of this method exceeds one of the receivers with the input ultra short impulses energy collection.

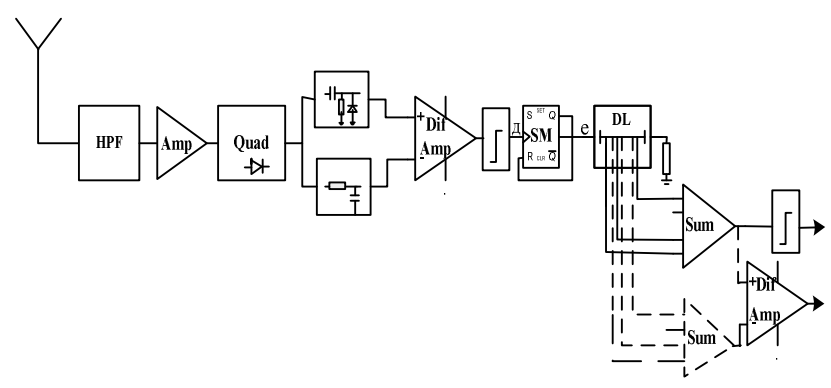

Fig.2 "Non energy" receiver circuit diagram: HPF - high pass filter, Amp - amplifier, 5 - limiter, Quad - quadrator, SM single shot multivibrator, Sum - summator.

\section{Information capacity of IR-UWB signals}

Usually each code sequence represents transmitted symbol and the address of the recipient. To provide simultaneous multi address transmissions in networks mutually orthogonal code sequences must be used. Such sequences are combinations of number of impulses of certain series with sufficient duty factor. This makes it possible to generate large orthogonal signal ensembles. But there is a contradiction: long impulse sequences with large number of impulses improve BER factor but limit data transmission rates and/or number of subscribers in the network utilizing ALOHA MAC protocols.

There is a method to increase data rates without data packet extension. In contrast to sinusoidal carrier, having two independent parameters - amplitude and phase, impulses have three - amplitude, duration and polarity. Modulation of these parameters separately or in a combination makes it possible to convert the address sequence, carrying one symbol, into the address sequence carrying $M=n \log _{2} k$ symbols, where $k$ - summary value of the impulses variable parameters gradations, $n-$ number of impulses in the address sequence. Thus, for example, with two gradations of amplitude, duration and polarity $(k=8)$, each impulse can carry 3 bits. An increase in the number of gradations (except polarity, which has only 2 values) makes it possible to increase information load on the signals. In our example, if $n=$ 1000, the address sequence carries 3000 bits. Fig. 4 shows vector diagram of impulse amplitude-durationpolarity modulation. 


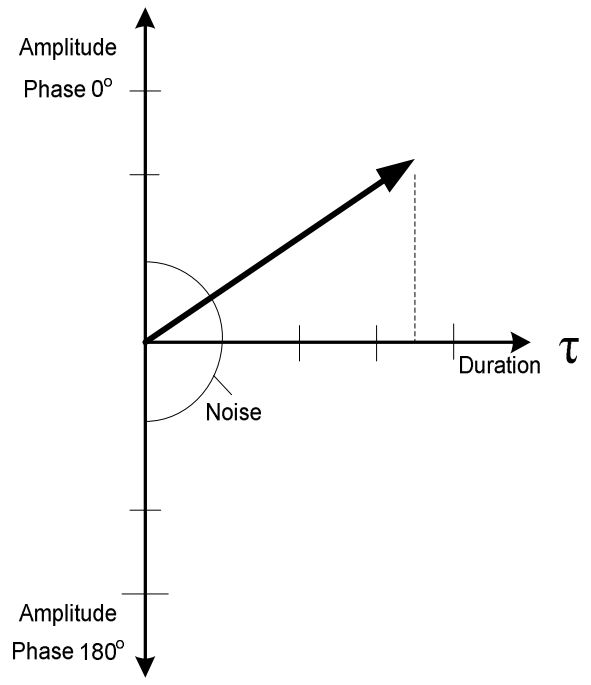

Fig. 4 Impulse modulation vector diagram

Receivers for such signals must be capable to recognize parameters of the impulses. It is not an easy task processing ultra short impulses.

\section{Conclusions}

Self-organizing ad hoc and MANET networks can occupy some important niches among modern communication systems. But up to now there are just a few such networks practically implemented. The main obstacle for this (among some others) is the multiple access control to communication channels especially with multi hop relaying. The problem can be solved by using IR-UWB signals in these networks. These signals permit to use uncontrolled multiple access with negligible probability of packet conflicts.
Yet another problem for implementation of IR-UWB signals in ad hoc and MANET networks is power and bandwidth limits imposed by FCC. To solve this obstacle new frequency bands can be used for these networks. We propose for this purpose frequencies band from 70 to $170 \mathrm{GHz}$. This band has relatively small atmospheric power losses, not crowded with radio services. Such wide frequency band makes it possible to have low power density of the signals to prevent possible interference to other radio services. Mutual interference protection can be significantly improved by using high pass filters on both IR-UWB transmitters and receivers.

A new method of "non energy" reception is proposed. The method simplifies ultra short impulse signals reception and processing.

To increase information capacity of IR-UWB signals impulse parameters modulation is proposed.

\section{References}

1. S. Bunin, A. Voiter, M. Ilchenko and V. Romanjuk, "Selforganized radio networks with ultra wideband signals", Naukova Dumka publication, Ukrainian Academy of Sciencies, Kyiv, 2012, 444 pp.

2. M. Ghravami, L.B. Michael and R. Kohno, "Ultra wide band signals and systems in communication engineering", John Wiley \& Sons, Ltd, Chichester, West Sussex, England, 248 pp.

3. FCC, "Revision of part 15 the commission rules regarding ultra-wideband transmittion systems", ET Docket, 2002.

4. S. Bunin, "Ultra short impulse radio signals in future long range radio communication systems", Proc. of 20-th International Conf. "SHF engineering and communications", Sevastopol, Crimea, Ukraine, Sept. 2010.

5. S. Bunin, "Non energetic" reception of ultra short impulse radio signals", Telecommunication Sciences, v.1, No.1, July December 2010, pp. 7 - 13 .

Received in final form April 23, 2014 